\title{
Quasiperiodic Floquet-Thouless Energy Pump
}

\author{
Frederik Nathan $\odot,{ }^{1,2}$ Rongchun Ge ${ }^{3}$ Snir Gazit $\odot,{ }^{4}$ Mark Rudner, ${ }^{1}$ and Michael Kolodrubetz $\oplus^{3}$ \\ ${ }^{1}$ Center for Quantum Devices, Niels Bohr Institute, University of Copenhagen, 2100 Copenhagen, Denmark \\ ${ }^{2}$ Department of Physics and Institute for Quantum Information and Matter, California Institute of Technology, \\ Pasadena, California 91125, USA \\ ${ }^{3}$ Department of Physics, The University of Texas at Dallas, Richardson, Texas 75080, USA \\ ${ }^{4}$ Racah Institute of Physics and The Fritz Haber Research Center for Molecular Dynamics, The Hebrew University of Jerusalem, \\ Jerusalem 91904, Israel
}

(Received 27 November 2020; accepted 9 September 2021; published 13 October 2021)

\begin{abstract}
We study a disordered one-dimensional fermionic system subject to quasiperiodic driving by two modes with incommensurate frequencies. We show that the system supports a topological phase in which energy is transferred between the two driving modes at a quantized rate. The phase is protected by a combination of disorder-induced spatial localization and frequency localization, a mechanism unique to quasiperiodically driven systems. We demonstrate that an analogue of the phase can be realized in a cavity-qubit system driven by two incommensurate modes.
\end{abstract}

DOI: 10.1103/PhysRevLett.127.166804

Periodic driving can be used as a tool for quantum control [1-14] and can even induce new phases of matter with no equilibrium analogues [15-30]. Recently, it was discovered that quasiperiodically driven systems also support their own unique phases of matter [31], despite having neither continuous nor discrete time-translation symmetry.

In this work, we report the discovery of a topological phase of matter in quasiperiodically driven systems. We study a one-dimensional (1D) fermionic system driven by two modes with incommensurate frequencies, $\omega_{1}$ and $\omega_{2}$. With spatial disorder, the system supports a phase which is characterized by quantized energy transport and a nonzero value of an integer-valued topological invariant $\nu$. When one end of the system is fully occupied by fermions while the other end is empty [as depicted in Fig. 1(a)], the system transfers energy between the driving modes at the quantized average rate $\nu \mathcal{P}_{0}$, where $\mathcal{P}_{0} \equiv \omega_{1} \omega_{2} / 2 \pi$ denotes the "quantum of energy transfer" (with $\hbar=1$ throughout). We refer to this phase as the quasiperiodic Floquet-Thouless energy pump (QFTEP).

The absence of time-translation symmetry gives the QFTEP features which have no analogue in equilibrium or Floquet systems. In particular, the QFTEP is protected by a combination of spatial and frequency localization [31-36], meaning the index $\nu$ can only change if this localization is destroyed. Here frequency localization is a phenomenon unique to quasiperiodically driven systems which arises only for sufficiently irrational $\omega_{2} / \omega_{1}$. The condition of irrational $\omega_{2} / \omega_{1}$ means the QFTEP features a fractally structured phase diagram (see Fig. 2 and discussion below for details).

The same "quantum of energy transfer" that we observe was recently encountered in Refs. [37-39]. In particular, Ref. [37] studied the same class of systems we consider here, in the limit where the second driving mode is adiabatic: $\omega_{2} \rightarrow 0$. For fine-tuned parameters, and in the absence of disorder, this system was shown to exhibit a similar quantized energy pumping phenomenon to that we observe here. Unlike the phenomena in Refs. [37-39], the QFTEP does not require adiabatic driving, is robust to disorder, and occupies a finite region of parameter space. Hence the QFTEP, in contrast to these earlier phenomena, constitutes a genuine phase of matter.

We propose an experimental realization of a dimensionally reduced version of the QFTEP in a two-level system (qubit) coupled to a quantized cavity mode and driven by

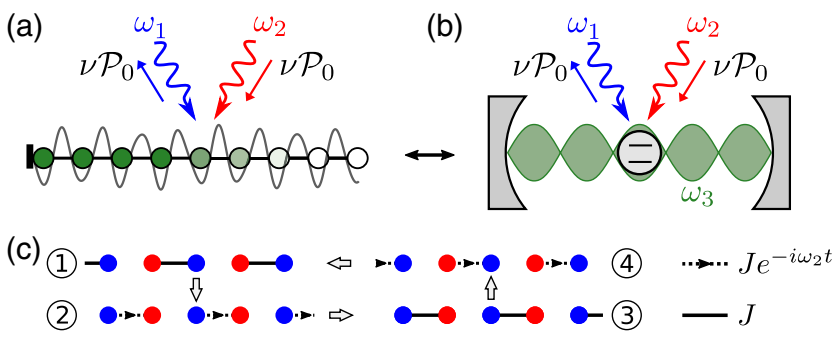

FIG. 1. (a) The quasiperiodic Floquet-Thouless energy pump for a fermionic chain driven by incommensurate frequencies $\omega_{1}$ and $\omega_{2}$. When one end of the chain is fully occupied (green), energy is pumped between the two modes at the rate $\nu \mathcal{P}_{0}$ $\left(\mathcal{P}_{0}=\omega_{1} \omega_{2} / 2 \pi\right)$, where $\nu \in \mathbb{Z}$ is the topological winding number of the phase. The quantization persists as long as the system is localized in both real and frequency space. (b) An analogue of the phase can be realized with a bichromatically driven qubit-cavity system. (c) 4-step driving protocol that realizes the phase. A particle tunneling along (against) the arrow acquires a phase $e^{-i \omega_{2} t}\left(e^{i \omega_{2} t}\right)$ 

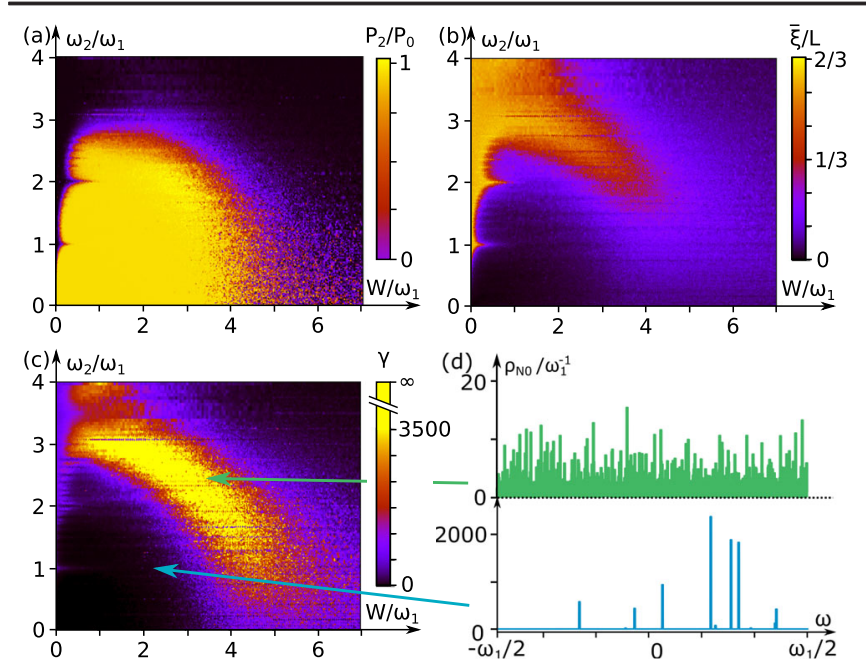

driving by two modes with incommensurate frequencies $\omega_{1}$ and $\omega_{2}$. Defining $\tilde{\mathcal{H}}\left(\phi_{1}, \phi_{2}\right) \equiv \hat{\mathcal{H}}_{0}\left(\phi_{1} / \omega_{1} ; \phi_{2}\right)$, the Hamiltonian can hence be written as $\hat{\mathcal{H}}(t)=\tilde{\mathcal{H}}\left(\omega_{1} t\right.$, $\left.\omega_{2} t\right)$, where $\tilde{\mathcal{H}}\left(\phi_{1}, \phi_{2}\right)$ is $2 \pi$-periodic in each of its arguments. Note that the discussion below applies to any quasiperiodically driven $1 \mathrm{D}$ system of noninteracting fermions whose Hamiltonian can be expressed in this form.

Because of the absence of interactions, time-evolved many-body states in the system can be resolved in terms of Slater determinants of time-evolved single-particle states. For simplicity, below we therefore consider the dynamics of the system with only a single particle present, unless otherwise stated. We use calligraphic symbols to denote many-body operators (acting in Fock space), and italic symbols for single-particle operators.

Frequency localization.-As a main result, this work

FIG. 2. Simulation of the QFTEP with 200 unit cells and open boundary conditions, as detailed in the numerics section. (a) Average energy absorption rate over 10000 periods of mode 1 when the left half of the chain is initially occupied. (b) Estimated spatial localization length of the generalized Floquet states, $\xi$. (c) Effective spectral density of the system, $\gamma$. (d) Spectral density $\rho_{N_{0}}(\omega)$ resulting from a wave packet initialized on a single site in the middle of the chain for parameters within the topological plateau (bottom), and at the transition (top).

two incommensurate frequencies [see Fig. 1(b)]. Our results indicate that such a simple physical setup inherits the topological properties of the QFTEP, suggesting the possibility of realizing this phase in cavity quantum electrodynamics.

Model.-Here we present a particular model that realizes the QFTEP. We consider a 1D bipartite tight-binding system with $L$ unit cells, with Hamiltonian $\hat{\mathcal{H}}_{0}(t ; \lambda)=\hat{\mathcal{H}}_{\mathrm{dr}}(t ; \lambda)+$ $\hat{\mathcal{H}}_{\text {dis }}$. Here, for fixed $\lambda \in \mathbb{R}, \hat{\mathcal{H}}_{\mathrm{dr}}(t ; \lambda)$ describes timedependent tunneling with period $T_{1}$, while $\hat{\mathcal{H}}_{\text {dis }}=$ $\sum_{x=1}^{2 L}\left(\Delta_{x} / 2\right) \hat{c}_{x}^{\dagger} \hat{c}_{x}$ describes a static on-site disorder potential. Here $\hat{c}_{x}$ annihilates a fermion on site $x$, while each $\Delta_{x}$ is picked randomly from the interval $[-W, W]$. We take the lattice constant to be 1 throughout. The driving term $\hat{\mathcal{H}}_{\mathrm{dr}}(t ; \lambda)$ is piecewise constant in $t$ over 4 steps of equal length [see Fig. 1(c)]. In step $s$, defined as the interval $[(s-1) / 4] T_{1} \leq$ $t<(s / 4) T_{1}, \hat{\mathcal{H}}_{\mathrm{dr}}(t ; \lambda)=-J \sum_{n=1}^{L}\left(e^{i \eta_{s} \lambda} \hat{c}_{2 n}^{\dagger} \hat{c}_{2 n+d_{s}}+\right.$ H.c. $)$, where $d_{1}=d_{4}=1, d_{2}=d_{3}=-1, \eta_{4}=-\eta_{2}=1$, and $\eta_{1}=\eta_{3}=0$. The parameter $\lambda$ controls the phases of the tunneling terms. We set $J=\left(2 \pi / T_{1}\right)$ such that $\hat{\mathcal{H}}_{\mathrm{dr}}(t ; \lambda)$ generates tunneling by precisely one site per step when $\lambda$ is fixed.

Related versions of the model above were studied in Refs. [16,22,37]. Reference [37] explored the case where $\lambda$ was increased adiabatically, and argued that this cyclic modulation caused a transfer of energy to the driving mode at the quantized rate of $\omega_{1}=2 \pi / T_{1}$ per cycle. In this work, we consider the case where $\lambda$ increases at a finite rate, $\lambda=\omega_{2} t$, such that the system is subject to quasiperiodic shows that the model above is characterized by an integervalued topological invariant when it is localized in the spatial and frequency domains. The key to understanding such localization is a generalized Floquet theorem [31-36]: for the bichromatically driven systems we consider, a complete orthonormal basis of generalized (single-particle) Floquet states $\left\{\left|\Phi_{n}\left(\phi_{1}, \phi_{2}\right)\right\rangle\right\}$ can be defined such that the time evolution of any state takes the form $|\psi(t)\rangle=$ $\sum_{n} \kappa_{n} e^{-i \varepsilon_{n} t}\left|\Phi_{n}\left(\omega_{1} t, \omega_{2} t\right)\right\rangle$. Here each $\left|\Phi_{n}\left(\phi_{1}, \phi_{2}\right)\right\rangle$ is $2 \pi$ periodic in each of its arguments while $\varepsilon_{n}$ is real valued and defines a generalized quasienergy. The structure above is equivalently captured in the single-particle evolution operator of the system, $\hat{\mathcal{U}}(t) \equiv \mathcal{T} e^{-i \int_{0}^{t} d t^{\prime} \hat{H}\left(t^{\prime}\right)}$, where $\mathcal{T}$ denotes the time-ordering operation and $\hat{H}(t)$ is the single-particle Hamiltonian of the system [i.e., $\hat{\mathcal{H}}(t)$ restricted to the oneparticle sector]. Specifically,

$$
\hat{U}(t)=\hat{P}\left(\omega_{1} t, \omega_{2} t\right) e^{-i \hat{H}_{\mathrm{eff}} t},
$$

where $\hat{P}\left(\phi_{1}, \phi_{2}\right) \equiv \sum_{n}\left|\Phi_{n}\left(\phi_{1}, \phi_{2}\right)\right\rangle\left\langle\Phi_{n}(0,0)\right|$ and $\hat{H}_{\text {eff }} \equiv$ $\sum_{n} \varepsilon_{n}\left|\Phi_{n}(0,0)\right\rangle\left\langle\Phi_{n}(0,0)\right|$ define a generalized micromotion operator and effective Hamiltonian for the system, respectively.

The decomposition in Eq. (1) is only useful if each generalized Floquet state $\left|\Phi_{n}\left(\phi_{1}, \phi_{2}\right)\right\rangle$ is a continuous function of $\phi_{1}$ and $\phi_{2}$, or, equivalently, if the twodimensional Fourier decomposition of $\left|\Phi_{n}\left(\phi_{1}, \phi_{2}\right)\right\rangle$ converges. This situation defines "frequency localization." With disorder, the generalized Floquet states may moreover be spatially localized [40], implying that particles remain confined near their initial location at all times. We refer to the combination of spatial and frequency localization as "full localization" below.

To infer the conditions for frequency localization, we work in the Fourier harmonic space corresponding to mode 2, yielding the Hamiltonian of an effective two-dimensional, periodically driven system, $\hat{\boldsymbol{H}}_{2 \mathrm{D}}(t)=\hat{\boldsymbol{H}}_{2 \mathrm{D}}\left(t+T_{1}\right)$. To this end we introduce a new degree of freedom, $\hat{n}_{2}$, whose 
corresponding "Fourier harmonic" Hilbert space is spanned by the states $\left\{|y\rangle_{F}, y \in \mathbb{Z}\right\}$, such that $\hat{n}_{2}|y\rangle_{F}=y|y\rangle_{F}$. Heuristically, $\hat{n}_{2}$ can be seen as counting the number of photons in mode 2 [41]. We obtain $\hat{\boldsymbol{H}}_{2 \mathrm{D}}(t)$ from $\hat{H}(t)$ by adding a term $\omega_{2} \hat{n}_{2}$ and replacing each phase factor $e^{i \omega_{2} t}$ in $\hat{H}(t)$ by $\sum_{y=-\infty}^{\infty}|y\rangle_{F}\left\langle y-\left.1\right|_{F}\right.$ (and similar for the corresponding Hermitian conjugate, $\left.e^{-i \omega_{2} t}\right)$. See Supplemental Material (SM) [40] for further details.

The Hamiltonian $\hat{\boldsymbol{H}}_{2 \mathrm{D}}(t)$ acts on the Hilbert space spanned by the states $|x, y\rangle\rangle \equiv|x\rangle \otimes|y\rangle_{F}$ where $y \in \mathbb{Z}$, $x=1,2,3, \ldots, 2 L$, and $|x\rangle$ denotes the single-particle state of the original 1D system with the particle located on site $x$. Thus, $\hat{\boldsymbol{H}}_{2 \mathrm{D}}(t)$ can be seen as the Hamiltonian of a two-dimensional lattice system whose sites are indexed by $x$ and $y$. Each Floquet state of $\left.\hat{\boldsymbol{H}}_{2 \mathrm{D}}(t),\left|\Psi_{n}(t)\right\rangle\right\rangle$, corresponds to a generalized Floquet state of $\hat{H}(t),\left|\Phi_{n}\left(\phi_{1}, \phi_{2}\right)\right\rangle$, via $\left\langle x \mid \Phi_{n}\left(\phi_{1}, \phi_{2}\right)\right\rangle=\sum_{y} e^{-i y \phi_{2}}\left\langle\left\langle x, y \mid \Psi_{n}\left(\phi_{1} / \omega_{1}\right)\right\rangle\right\rangle[32,40]$. The $y$ dependence of $\left.\left|\Psi_{n}(t)\right\rangle\right\rangle$ thus encodes the Fourier components of $\left|\Phi_{n}\left(\phi_{1}, \phi_{2}\right)\right\rangle$ with respect to $\phi_{2}$. Hence, full localization corresponds to localization of the Floquet states of $\hat{\boldsymbol{H}}_{2 \mathrm{D}}(t)$ due to Anderson localization in the spatial direction and Wannier-Stark localization in the frequency (Fourier harmonic) direction [40].

The above considerations imply that frequency localization requires irrational $\omega_{2} / \omega_{1}$ : when $\omega_{2} / \omega_{1}$ is sufficiently close to $p / q$ for some integers $p$ and $q$, the oscillating terms of $\boldsymbol{H}_{2 \mathrm{D}}(t)$ resonantly couple sites separated by $q$ lattice constants in the $y$ direction, inducing $y$ delocalization, and hence frequency delocalization after translation back to the Hilbert space of the physical (1D) problem at hand $[31,35,40]$. We thus expect frequency localization to break down in some $\omega_{2}$ interval around $p \omega_{1} / q$ for each choice of integers $p$ and $q$. However, the width of this interval may decrease with increasing $p$ and $q$, allowing frequency localization to occur for a finitemeasure set of $\omega_{2}$ [40].

Topological invariant.- The topological invariant of the QFTEP can be defined from the generalized micromotion operator in Eq. (1). For simplicity, we consider a system with periodic boundary conditions; the results can be applied directly to systems with open boundary conditions. We first define a "phase-twisted" micromotion operator $\hat{P}_{\theta}\left(\phi_{1}, \phi_{2}\right)$ by adding a factor $e^{-i \theta}\left(e^{i \theta}\right)$ to the matrix elements of $\hat{P}\left(\phi_{1}, \phi_{2}\right)$ that transfer a particle across an arbitrary reference bond $x_{0}$ in the positive (negative) $x$ direction [40]. When the system is fully localized, $\hat{P}_{\theta}\left(\phi_{1}, \phi_{2}\right)$ is unitary, as well as continuous and periodic in $\theta, \phi_{1}$, and $\phi_{2}$ [40]. Under these conditions, $\hat{P}_{\theta}\left(\phi_{1}, \phi_{2}\right)$ is characterized by an integer valued winding number:

$\nu=\oint \frac{d \theta d^{2} \phi}{8 \pi^{2}} \operatorname{Tr}\left(\hat{P}_{\theta}^{-1} \partial_{\theta} \hat{P}_{\theta}\left[\hat{P}_{\theta}^{-1} \partial_{\phi_{2}} \hat{P}_{\theta}, \hat{P}_{\theta}^{-1} \partial_{\phi_{1}} \hat{P}_{\theta}\right]\right)$,

where $d^{2} \phi=d \phi_{1} d \phi_{2}$, and we suppressed the phase dependence of $\hat{P}_{\theta}\left(\phi_{1}, \phi_{2}\right)$ for brevity. The index $\nu$ cannot change under smooth deformations of the system parameters that preserve full localization, and thus defines the invariant of the QFTEP. Nonzero values of $\nu$ can arise for weak or moderate disorder, where particles undergo nontrivial micromotion while their dynamics remain localized on long length scales.

The invariant $\nu$ can be seen as a dimensional reduction of the winding number of the anomalous Floquet-Anderson insulator (AFAI) [22,23]. Recall that full localization of $\hat{H}(t)$ occurs when the Floquet eigenstates of $\hat{\boldsymbol{H}}_{2 \mathrm{D}}(t)$ are localized. In this case, $\hat{\boldsymbol{H}}_{2 \mathrm{D}}(t)$ is characterized by the integer-valued winding number of the AFAI $[22,23]$. A straightforward derivation shows that this winding number is identical to $\nu$ [40].

Bulk-edge correspondence.-For a system with open boundary conditions, a nonzero value of $\nu$ implies a quantized transport of energy between modes 1 and 2 when all sites near one edge are occupied. Using the correspondence between Floquet states of $\hat{\boldsymbol{H}}_{2 \mathrm{D}}(t)$ and generalized Floquet states of $\hat{H}(t)$, in the SM we show that the time-averaged rate of work done on mode 2 , $\hat{\mathcal{P}}_{2}(t) \equiv-\omega_{2} \partial_{\phi_{2}} \tilde{\mathcal{H}}\left(\omega_{1} t, \omega_{2} t\right)$, is quantized when the system initially has sites $1, \ldots x_{1}$ occupied, for some $x_{1}$ in the bulk of the chain:

$$
\lim _{\tau \rightarrow \infty} \frac{1}{\tau} \int_{0}^{\tau} d t\left\langle\hat{\mathcal{P}}_{2}(t)\right\rangle=\nu \mathcal{P}_{0} .
$$

Conservation of energy dictates that the average rate of work done on mode 1 is given by $-\nu \mathcal{P}_{0}$. Since Eq. (3) is independent of $x_{1}$, only fermions near the edge contribute to the quantized energy transfer above. This result establishes the bulk-edge correspondence of the QFTEP.

To understand Eq. (3), note that for a finite open chain, $\hat{\boldsymbol{H}}_{2 \mathrm{D}}(t)$ describes an AFAI on an infinite strip in the $y$ direction (corresponding to "photon number" of mode 2). The bulk-edge correspondence for the AFAI dictates that $\hat{\boldsymbol{H}}_{2 \mathrm{D}}(t)$ supports chiral modes propagating along the edges of the strip that carry a quantized average current $\nu / T_{1}$ and $-\nu / T_{1}$ along the $y$ direction at the left and right end of the strip, respectively [22]. According to the mapping through which $y$ corresponds to the number of photons absorbed by mode 2, we expect that particles on the left edge transfer energy to mode 2 at the quantized average rate $\nu \mathcal{P}_{0}$.

The quantized energy transfer to mode 2 is supported by topologically protected edge modes: through the relationship between the QFTEP and AFAI established above, the existence of chiral edge states in the AFAI implies that each end of the QFTEP supports a family of generalized Floquet states which are localized spatially but delocalized in frequency space. These families of states, or "edge modes," are topologically protected features that can only disappear if full localization is destroyed in the bulk. When all states in one such topological edge mode are occupied, they collectively generate a quantized flow of energy to mode 2 
at the rate $\pm \nu \mathcal{P}_{0}$. Away from full occupation, we expect quantization persists if the particle density, $p(x)$, is locally uniform over the characteristic localization length scale of generalized Floquet states [42]. In this case, the left and right topological edge modes are occupied with probability $p(0)$ and $p(L)$, respectively, resulting in the energy transfer rate $\nu \mathcal{P}_{0}[p(0)-p(L)]$.

Numerical simulations. - We demonstrate the quantized energy pumping of the QFTEP through numerical simulations of the model presented in the introduction. We simulated a system of $L=200$ unit cells (400 sites) with open boundary conditions, initialized by only filling the leftmost 200 sites with fermions. Using direct time evolution, we computed the time-averaged rate of energy transfer to mode $2, \overline{\mathcal{P}}_{2}$, by averaging $\left\langle\hat{\mathcal{P}}_{2}(t)\right\rangle$ over the time interval from 0 to $t_{\mathrm{f}}=10000 T_{1}$. Figure 2(a) shows $\overline{\mathcal{P}}_{2} / \mathcal{P}_{0}$, as a function of $\omega_{2}$ and $W$ [43]. The data indicate a large plateau of quantized energy pumping $\left(\overline{\mathcal{P}}_{2}=\mathcal{P}_{0}\right)$ at finite values of $\omega_{2}$ and $W$, supporting our conclusions above.

The data in Fig. 2(a) exhibit several features that are consistent with our discussion above. The plateau with $\overline{\mathcal{P}}_{2}=\mathcal{P}_{0}$ has maximal extent along the $\omega_{2}$ direction when $W \approx \omega_{1}$, indicating that weak to moderate disorder stabilizes the QFTEP (interestingly, for $\omega_{2} \ll \omega_{1}$, stabilization occurs for very weak disorder). The quantized plateau diminishes when $\omega_{2} / \omega_{1}$ approaches values $p / q$ for integers $p$ and $q$, as is particularly clear for $q=1$, where the yellow plateau region is sharply "pinched in." In the SM we provide high-resolution data confirming that quantization of $\overline{\mathcal{P}}_{2}$ breaks down for other values of $p$ and $q$, with the breakdown most pronounced for smaller $p$ and $q$. These data thus support our prediction that the QFTEP is protected by the irrationality of $\omega_{2} / \omega_{1}$.

To characterize the phase transitions of the QFTEP, we estimate the spatial localization length of the generalized Floquet states in the system via $\xi \equiv$ $\sup _{x_{1}}\left(\sum_{x_{2}}\left|\left\langle x_{1}\left|\hat{U}\left(t_{\mathrm{f}}\right)\right| x_{2}\right\rangle\right|^{2}\left(x_{1}-x_{2}\right)^{2}\right)^{1 / 2}$. Figure 2(b) shows $\xi$ for the same parameters as taken in Fig. 2(a). For $W \lesssim 4 \omega_{1}$, the boundary of the topological plateau in Fig. 2(a) clearly coincides with the region in Fig. 2(b) where $\xi \sim \mathcal{O}(L)$ (indicating delocalization). For $\omega_{2}, W \gtrsim$ $3 \omega_{1}$ we observe a localized, topologically trivial phase with $\xi \sim \mathcal{O}(1)$ and $\overline{\mathcal{P}}_{2}=0$ [Figs. 2(a) and 2(b)]. Rather than a direct transition to this topologically trivial phase, Fig. 2(b) indicates the existence of an intermediate delocalized region for $W \lesssim 3 \omega_{1}, \omega_{2} \gtrsim 3 \omega_{1}$.

When $W$ exceeds $3 \omega_{1}$, the topological phase transition changes its qualitative behavior: $\xi$ decreases and $\overline{\mathcal{P}}_{2}$ displays irregular fluctuations for a finite $\omega_{2}$ interval. To investigate the transition here, we computed a measure of the frequencyspace localization length, using the time evolution of a single-particle state initialized on a particular site $x_{\mathrm{i}}$ in the chain, $|\psi(t)\rangle \equiv \hat{U}(t)\left|x_{\mathrm{i}}\right\rangle$. For a given (large) integer $N$, we let $\left|\psi_{N}(\omega)\right\rangle \equiv\left(1 / \sqrt{\omega_{1} N}\right) \sum_{n=1}^{N} e^{i n \omega T_{1}}\left|\psi\left(n T_{1}\right)\right\rangle \quad$ denote the finite-time discrete Fourier transform of $|\psi(t)\rangle$ when sampled stroboscopically with the period of mode 1 . We then define an effective spectral density $\gamma$ characterizing $|\psi(t)\rangle$ via the inverse participation ratio of the normalized spectral distribution $\rho_{N}(\omega) \equiv\left|\left\langle\psi_{N}(\omega) \mid \psi_{N}(\omega)\right\rangle\right|$ : $\gamma \equiv\left(1 / \omega_{1}\right) \lim _{N \rightarrow \infty} N /\left[\int_{0}^{\omega_{1}} d \omega \rho_{N}(\omega)^{2}\right]$. When $|\psi(t)\rangle$ has a dense Fourier spectrum, $\int_{0}^{\omega_{1}} d \omega \rho_{N}(\omega)^{2}$ remains finite in the limit $N \rightarrow \infty$, and thus $\gamma$ diverges. However, for a discrete spectrum, where $\rho_{\infty}(\omega) \equiv \lim _{N \rightarrow \infty} \rho_{N}(\omega)$ is given by a discrete sum of delta functions, the integral diverges linearly with $N$, such that $\gamma$ remains finite. In this case, $\gamma$ gives the inverse sum of the squared peak weights in $\rho_{\infty}(\omega)$; i.e., it measures the effective number of peaks in the frequency spectrum of $|\psi(t)\rangle$ (modulo $\omega_{1}$ ), $\rho_{\infty}(\omega)$. We hence expect that $\gamma$ is a good proxy for the localization length in the frequency space of mode 2 .

Figure 2(c) shows the maximal value of $\gamma$ obtained from 15 time-evolved single-particle states with initial positions $x_{\mathrm{i}}$ randomly chosen within the middle third of the system. We used the same parameters as considered in panels (a) and (b), and set $N$ to $N_{0}=10000$. As an illustration, in Fig. 2(d), we plot $\rho_{N_{0}}(\omega)$ for two parameter sets [indicated with arrows in Fig. 2(c)] where $\gamma$ is large (upper) and small (lower), respectively. Although Fig. 2(b) does not conclusively indicate whether spatial delocalization is present for $W \gtrsim 3 \omega_{1}$, Fig. 2(c) shows that the system undergoes frequency delocalization throughout the entire topological phase boundary.

Realization in a driven two-level system.-Here we propose a dimensionally reduced experimental realization of the QFTEP in a two-level system (qubit) driven by three incommensurate modes. The model presented in the introduction is mapped to this platform by taking the limit of zero disorder, and replacing spatial crystal momentum with the phase of a third driving mode: $e^{i k} \rightarrow e^{i \omega_{3} t}$ [44]. When the frequencies $\omega_{1}, \omega_{2}$, and $\omega_{3}$ are sufficiently incommensurate, the generalized Floquet eigenstates of the system remain localized due to the quasidisorder of the lattice in the three-dimensional frequency space [3136,40]. The analysis below Eq. (1) thereby also yields the topological index $\nu$ for this model, defined by Eq. (2) with $\theta$ replaced by the phase $\phi_{3}=\omega_{3} t$ of the third driving field. In the SM we provide data from numerical simulations that confirm the three-mode driven qubit model described above supports a topologically nontrivial regime characterized by $\nu=1$. Edges can naturally be incorporated into the qubit realization by replacing one or more of the driving modes by quantized cavity modes, whose vacuum states define a natural edge.

Summary and outlook.-This work establishes the QFTEP as a new nonequilibrium topological phase. The QFTEP elevates the Floquet-Thouless energy pump (FTEP) in Ref. [37] from a fine-tuned (but nonetheless interesting) phenomenon to a genuine stable phase of matter. Whereas the FTEP requires adiabatic driving, 
fine-tuning, and is formally destroyed by disorder [37], the QFTEP arises for finite $\omega_{1}$ and $\omega_{2}$, and is stabilized by disorder. In a setting with open boundary conditions, the QFTEP is characterized by robust, quantized energy pumping between the two driving modes, supported by topological edge states.

We showed that a qubit driven by three incommensurate modes can realize a dimensionally reduced version of the QFTEP. Such a system is a promising platform for experimental realization of this phenomenon due to welldeveloped techniques for controlling and driving qubits [45]. The QFTEP may also be directly realized in onedimensional quantum chains, such as systems of ultracold atoms in optical lattices, or trapped-ion systems. The robustness to disorder and finite modulation frequency makes such realizations of the QFTEP more feasible than for the adiabatic FTEP.

Quantization of energy pumping breaks down for rational values of $\omega_{1} / \omega_{2}$, implying that the phase diagram of the QFTEP has a fractal structure. Understanding this fractal structure and its role for the phase transitions of the QFTEP will be an interesting direction for future studies.

Another prospect for investigations is to apply our dimensional reduction scheme to other equilibrium or Floquet topological phases of matter. A more complete investigation of the physical signatures of the QFTEP, its stability to interactions, and its place in the expanding classification of non-equilibrium topological phases of matter will also be important future directions.

We thank Anushya Chandran, Philip Crowley, David Long, Ivar Martin, and Gil Refael for valuable discussions. This work was performed with support from the National Science Foundation through Grant No. DMR-1945529 and the Welch Foundation through Grant No. AT-203620200401 (M. K. and R. G.). S. G. acknowledges support from the Israel Science Foundation, Grant No. 1686/18. F. N. and M.R. gratefully acknowledge the support of the European Research Council (ERC) under the European Union Horizon 2020 Research and Innovation Programme (Grant Agreement No. 678862), and the Villum Foundation. We used the computational resources of the Lonestar 5 cluster operated by the Texas Advanced Computing Center at the University of Texas at Austin and the Ganymede and Topo clusters operated by the University of Texas at Dallas' Cyberinfrastructure \& Research Services Department.

Note added.-Recently, a preprint appeared which describes an energy pumping phenomenon related to the one we consider here [46], in the context of a general classification. Our work is fully consistent with Ref. [46], and provides a complementary perspective on the phenomenon, including a study of the role of spatial disorder, along with additional experimental proposals for realizing the phase.
[1] L. M. K. Vandersypen and I. L. Chuang, Rev. Mod. Phys. 76, 1037 (2005).

[2] M. Bukov, L. D’Alessio, and A. Polkovnikov, Adv. Phys. 64, 139 (2015).

[3] P. Weinberg, M. Bukov, L. D’Alessio, A. Polkovnikov, S. Vajna, and M. Kolodrubetz, Phys. Rep. 688, 1 (2017).

[4] N. H. Lindner, G. Refael, and V. Galitski, Nat. Phys. 7, 490 (2011).

[5] J. Struck, C. Ölschläger, M. Weinberg, P. Hauke, J. Simonet, A. Eckardt, M. Lewenstein, K. Sengstock, and P. Windpassinger, Phys. Rev. Lett. 108, 225304 (2012).

[6] Y. H. Wang, H. Steinberg, P. Jarillo-Herrero, and N. Gedik, Science 342, 453 (2013).

[7] G. Jotzu, M. Messer, R. Desbuquois, M. Lebrat, T. Uehlinger, D. Greif, and T. Esslinger, Nature (London) 515, 237 (2014).

[8] J. W. McIver, B. Schulte, F. U. Stein, T. Matsuyama, G. Jotzu, G. Meier, and A. Cavalleri, Nat. Phys. 16, 38 (2020).

[9] L. Viola and S. Lloyd, Phys. Rev. Lett. 58, 2733 (1998).

[10] L. Viola, E. Knill, and S. Lloyd, Phys. Rev. Lett. 82, 2417 (1999).

[11] G. de Lange, A. H. Wang, D. Risté, V. V. Dobrovitski, and R. Hanson, Science 330, 60 (2010).

[12] J. Dalibard, F. Gerbier, G. Juzeliunas, and P. Öhberg, Rev. Mod. Phys. 83, 1523 (2011).

[13] J. Struck, C. Ölschläger, R. Le Targat, P. Soltan-Panahi, A. Eckardt, M. Lewenstein, P. Windpassinger, and K. Sengstock, Science 333, 996 (2011).

[14] N. Goldman and J. Dalibard, Phys. Rev. X 4, 031027 (2014).

[15] L. Jiang, T. Kitagawa, J. Alicea, A. R. Akhmerov, D. Pekker, G. Refael, J. I. Cirac, E. Demler, M. D. Lukin, and P. Zoller, Phys. Rev. Lett. 106, 220402 (2011).

[16] M. S. Rudner, N. H. Lindner, E. Berg, and M. Levin, Phys. Rev. X 3, 031005 (2013).

[17] F. Nathan and M.S. Rudner, New J. Phys. 17, 125014 (2015).

[18] V. Khemani, A. Lazarides, R. Moessner, and S. L. Sondhi, Phys. Rev. Lett. 116, 250401 (2016).

[19] von Keyserlingk C. W. and S. L. Sondhi, Phys. Rev. B 93, 245145 (2016).

[20] von Keyserlingk C. W. and S. L. Sondhi, Phys. Rev. B 93, 245146 (2016).

[21] T. Kitagawa, E. Berg, M. Rudner, and E. Demler, Phys. Rev. B 82, 235114 (2010).

[22] P. Titum, E. Berg, M. S. Rudner, G. Refael, and N. H. Lindner, Phys. Rev. X 6, 021013 (2016).

[23] F. Nathan, M. S. Rudner, N. H. Lindner, E. Berg, and G. Refael, Phys. Rev. Lett. 119, 186801 (2017).

[24] F. Nathan, D. Abanin, E. Berg, N. H. Lindner, and M. S. Rudner, Phys. Rev. B 99, 195133 (2019).

[25] K. Wintersperger, C. Braun, F. N. Ünal, A. Eckardt, M. D. Liberto, N. Goldman, I. Bloch, and M. Aidelsburger, Nat. Phys. 16, 1058 (2020).

[26] K. Sacha, Phys. Rev. A 91, 033617 (2015).

[27] D. V. Else, B. Bauer, and C. Nayak, Phys. Rev. Lett. 117, 090402 (2016).

[28] N. Y. Yao, A.C. Potter, I.-D. Potirniche, and A. A. Vishwanath, Phys. Rev. Lett. 118, 030401 (2017). 
[29] J. Zhang, P. W. Hess, A. Kyprianidis, P. Becker, A. Lee, J. Smith, G. Pagano, I.-D. Potirniche, A. C. Potter, A. Vishwanath, N. Y. Yao, and C. Monroe, Nature (London) 543, 217 (2017).

[30] S. Choi, J. Choi, R. Landig, G. Kucsko, H. Zhou, J. Isoya, F. Jelezko, S. Onoda, H. Sumiya, V. Khemani, C. Keyserlingk, N. Y. Yao, E. Demler, and M. D. Lukin, Nature (London) 543, 221 (2017).

[31] D. V. Else, W. W. Ho, and P. T. Dumitrescu, Phys. Rev. X 10, 021032 (2020).

[32] T.-S. Ho, S.-I. Chu, and J. V. Tietz, Chem, Phys. Lett. 96, 464 (1983).

[33] J. M. Luck, H. Orland, and U. Smilansky, J. Stat. Phys. 53, 551 (1988).

[34] G. Casati, I. Guarneri, and D. L. Shepelyansky, Phys. Rev. Lett. 62, 345 (1989).

[35] P. M. Blekher, H. R. Jauslin, and J. L. Lebowitz, J. Stat. Phys. 68, 271 (1992).

[36] H. Hatami, C. Danieli, J. D. Bodyfelt, and S. Flach, Phys. Rev. E 93, 062205 (2016).

[37] M. H. Kolodrubetz, F. Nathan, S. Gazit, T. Morimoto, and J. E. Moore, Phys. Rev. Lett. 120, 150601 (2018).
[38] I. Martin, G. G. Refael, and B. Halperin, Phys. Rev. X 7, 041008 (2017).

[39] F. Nathan, I. Martin, and G. Refael, Phys. Rev. B 99, 094311 (2019).

[40] See Supplemental Material at http://link.aps.org/ supplemental/10.1103/PhysRevLett.127.166804 for additional data from numerical simulations and details of derivations.

[41] J. H. Shirley, Phys. Rev. 138, B979 (1965).

[42] B. Ye, F. Machado, C. D. White, R. S. K. Mong, and N. Y. Yao, Phys. Rev. Lett. 125, 030601 (2020).

[43] Each value of $\omega_{2}$ we probed was obtained by adding a small random number to a value generated by a different algorithm. Consequently, each sampled value of $\omega_{2}$ was incommensurate with $\omega_{1}$ (up to machine precision) for all but a measure-zero set of outcomes.

[44] X.-L. Qi, T. L. Hughes, and S.-C. Zhang, Phys. Rev. B 78, 195424 (2008).

[45] K. J. Vahala, Nature (London) 424, 839 (2003).

[46] D. M. Long, P. J. D. Crowley, and A. Chandran, Phys. Rev. Lett. 126, 106805 (2021). 